\title{
Vision-based monitoring of heritage monuments: Unmanned Aerial Systems (UAS) for detailed inspection and high-accuracy survey of structures
}

\author{
N. Hallermann ${ }^{1}$, G. Morgenthal ${ }^{1} \&$ V. Rodehorst ${ }^{2}$ \\ ${ }^{1}$ Modelling and Simulation of Structures, Institute of Structural \\ Engineering, Bauhaus-Universität Weimar, Germany \\ ${ }^{2}$ Computer Vision in Engineering, \\ Bauhaus-Universität Weimar, Germany
}

\begin{abstract}
The maintenance and preservation of heritage structures is of particular importance from a historical, cultural, socio-political, economic and not least architectural and aesthetic point of view. An efficient maintenance scheme requires periodical inspections associated with a reliable condition determination of the structure. Especially the process of data acquisition is a time consuming, labour intensive and technically complex task. This paper presents a vision-based inspection and monitoring approach for heritage structures based on high quality aerial photos taken by remote controlled Unmanned Aerial Systems (UAS). In terms of quality, time and investment this approach provides an important contribution to data acquisition processes and monitoring strategies and will provide an increased efficiency in preserving architectural heritages. UAS's equipped with high quality photo or video cameras open up the possibility of simplifying and accelerating data acquisition and monitoring tasks. The extreme flight stability of the UAS and the fully compensated camera provide high quality data which are essential for reliable condition monitoring and inspection results. This also offers the possibility of highly accurate survey and geo-referenced 3Dmodelling of structures in relation to direct referenced visualisation of detected damages. The paper shows three case studies. Furthermore, it presents a combination of conventional inspection measurements with modern
\end{abstract}


photogrammetric and computer vision methods for an automatic post-flight georeferenced 3D reconstruction based on aerial images.

Keywords: monitoring, survey, uas, uav, heritage monuments, inspection, computer vision, 3D-modelling, damage detection, non-destructive testing.

\section{Introduction}

The preservation of heritage monuments such as cathedrals, castles or special parts of historical buildings like sculptures is a specifically complex task for civil engineers, architects, restaurateurs and conservators. In regard to historical, cultural, socio-political, economic and not least architectural and aesthetic issues the maintenance and preservation of heritage structures is of particular importance. An efficient maintenance requires for regular inspections associated with a reliable state determination of the structure. The principal part of conventional inspections is based on visual investigations which have to be performed by experts, who often document their investigations by drawings or photos. This process of data acquisition and documentation is a time consuming, labour intensive and technically complex task. Particularly in the case of structures or elements that are difficult to access, special scaffolding, elevating platforms or specially trained climbers are required for data acquisition. This can lead to long term and cost intensive inspections as well as to constraints in usability and aesthetics of the building.

This paper presents a vision-based inspection and monitoring approach for heritage structures based on high quality aerial photos taken by remote controlled Unmanned Aerial Systems (UAS). In terms of quality, time and investment this approach provides an important contribution to data acquisition processes, existing monitoring strategies and will ensure an increased efficiency of preserving architectural heritages. UAS's equipped with high quality photo or video cameras open up the possibility of simplifying and accelerating data acquisition and monitoring tasks. Supported by Global Positioning System and other special features like an altitude controlled flight mode UAS can be used for nondestructive outdoor and indoor data acquisition in close proximity to sensitive structures. The extreme flight stability of the UAS and the fully compensated camera guarantee high quality data which are essential for reliable condition determinations and inspection results. High quality images offer also the possibility of high-accuracy survey and geo-referenced dense 3D-reconstruction of structures in relation to direct referenced visualisation of deformations or damages. In three case studies the paper shows the workflow of UAS-based data acquisition on heritage structures and the potential of high quality data for the condition determination and monitoring of structures. Furthermore, it presents a combination of conventional inspection measurements with modern photogrammetric and computer vision methods for a post-flight geo-referenced $3 \mathrm{D}$ reconstruction based on aerial images. 


\section{Unmanned Aerial Systems (UAS): applications, limits and flight system configuration}

UAS have improved rapidly in the last years and become more and more important for civil applications. These systems can be used for applications in the field of professional airborne photo- and videography, geology, geography, in agriculture or forestry and especially in the field of civil engineering and surveillance for the inspection of structures. The investigations and case studies presented here focus on vision-based condition determination and monitoring of heritage monuments, particularly on aerial image based visual inspection and high accurate survey of structures. Visual based inspection or image based surveillance tasks could extensively simplified with these very small (less than $5 \mathrm{~kg}$ ), remote controlled and electrically operating flight systems. These flight systems can be equipped with different kinds of high definition photo, video or infrared cameras. The variety of applications in civil engineering was already shown in different case studies like [1-5]. In spite of all benefits, UAS's have some technical and legal limitations, which confine the use of these systems. Due to the small payload, only light digital compact cameras can be used for photo or video data acquisition. Furthermore, the limited payload allows only small battery packs, which leads to relatively short operating times. Due to the low weight, the flight systems react very sensitively in critical wind situations. Unexpected critical flight situations or flight missions without a supporting GPS-signal require a well-trained pilot. A sensor based active and robust collision avoidance is currently in development test phase. Further limits are given by the regulations of civil and security authorities, e.g. flight permissions, altitude limits or the restrictions for fully autonomous flights.

\subsection{Hardware and software features}

The flight system used for the investigations and case studies on heritage structures shown here was a Falcon 8 developed and patented by Ascending Technologies (Germany) [6], shown in fig. 1.

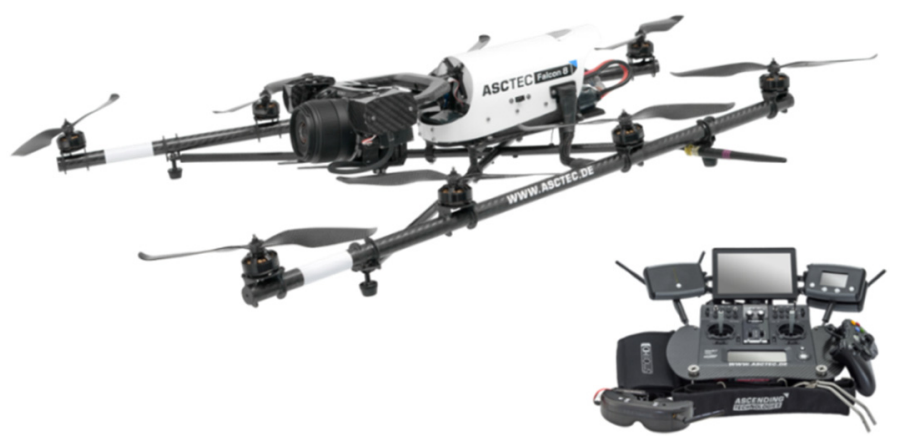

Figure 1: Flight system Falcon 8 (Ascending Technologies) of BauhausUniversät Weimar with Sony Alpha 7R and ground control station. 
This professional, very light (take-off weight $2.2 \mathrm{~kg}$ ) high-end multi rotor platform with eight rotors, which are arranged on two arms in V-shape configuration, is an extremely wind stable flight system, for flights up to wind speeds of $15 \mathrm{~m} / \mathrm{s}$. The central flight control unit with the fully actively stabilised and compensated camera mount is placed in the centroid between both motor carrying arms, which allows for a horizontal angular freedom of $360^{\circ}$ and a camera tilt of $90^{\circ}$ up and down. This guarantees a unique field of view. The highly accurate stabilisation, positioning and orientation of the flight system is realised by a high frequency triple redundant computing unit Autopilot Trinity. The extreme position accuracy and stability, the redundancy of each motor-rotor combination and the Autopilot Trinity guarantee a maximum of safety and allow for complex outdoor and also indoor flight missions. With a payload of $750 \mathrm{~g}$, it is possible to use high quality compact digital photo, video or infrared cameras. The specifications of the used camera equipment are summarised in table 1.

Table 1: Specifications of high quality digital photo and video cameras.

\begin{tabular}{|l|l|l|}
\hline Specification & $\begin{array}{l}\text { Sony Alpha 7R } \\
\text { (SLR-style mirrorless) }\end{array}$ & $\begin{array}{l}\text { Sony Handycam Camcorder } \\
\text { HDR-PJ810E }\end{array}$ \\
\hline Resolution & 36 megapixels $(7360$ x 4912) & 24 megapixels \\
\hline Sensor & CMOS Full frame $(35.9 \times 24 \mathrm{~mm})$ & $\begin{array}{l}\text { Exmor R CMOS Sensor }(T y p \\
1 / 3.95 / 4.6 \mathrm{~mm})\end{array}$ \\
\hline Zoom & Prime lens (interchangeable) & $12 \mathrm{x}$ optical zoom \\
\hline Lens & $\begin{array}{l}\text { Zeiss Sonnar T FE } 35 \mathrm{~mm} \text { F2,8 ZA } \\
\text { Normal lens 35 mm }\end{array}$ & G-Lens 26.8-321.6 mm (3 mm) \\
\hline $\begin{array}{l}\text { Image } \\
\text { Stabiliser }\end{array}$ & No & $\begin{array}{l}\text { Balanced Optical SteadyShot with } \\
\text { active mode }\end{array}$ \\
\hline Video & $1920 \mathrm{x} 1080 / 60 \mathrm{p}$ & $1920 \mathrm{x} 1080 / 50 \mathrm{p}$ \\
\hline
\end{tabular}

The flight system and also the mounted camera can be controlled respectively triggered remotely with the mobile ground station. For team operation in complex flight missions the camera can be controlled by a second operator independently with an additional control pad. Camera settings, camera tilt and orientation can be easily changed during the flight. For compensating movements of the flight system the camera is continuously levelled horizontally (roll stabilised) and vertically (pitch stabilised). High performance battery packs allow flights of up to 14 minutes, which is sufficient for most of the applications.

\section{Vision-based monitoring of heritage monuments}

The application of Unmanned Aerial Systems for the inspection and monitoring of heritage monuments were investigated in a research project [7] in cooperation with the developer of the flight systems and various experts (cathedral architects, restaurateurs and conservators) in the field of care and preservation of historical monuments. The project focused on the condition determination of structures 
based on high quality images and videos. The usability and efficiency of this method was investigated on different reference objects by performing intensive practical tests. A small part of the investigations and results is presented in the following three case studies.

\subsection{Aerial image based visual damage detection}

The application of detailed visual inspection based on high quality aerial images and videos was investigated on the 1200 years old Halberstadt Cathedral, Germany, shown in fig. 2.

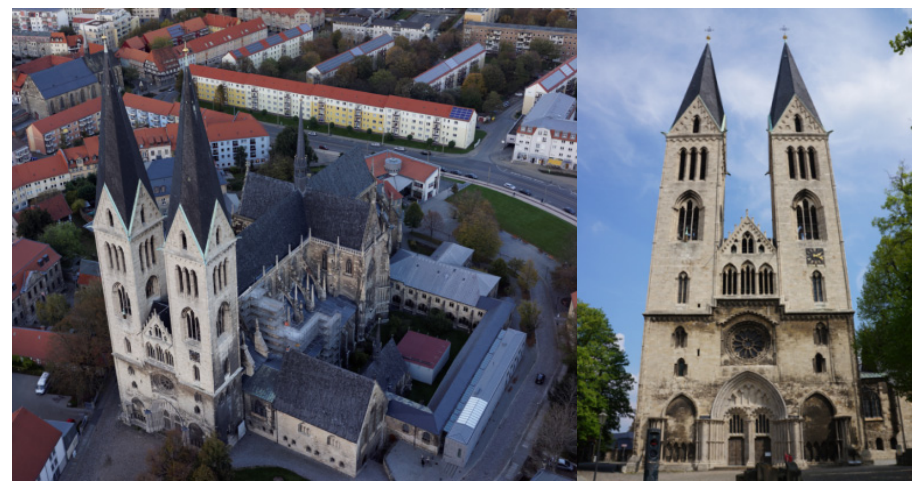

Figure 2: Aerial photo (southwest view) and ground photo (west view) of Halberstadt Cathedral.

This cathedral with the $102 \mathrm{~m}$ long nave and the two $91 \mathrm{~m}$ high spires was built in gothic style and is situated in the historical centre of the city. The scope of investigations was split into outdoor and indoor and also in still image and livevideo inspections, focussed on the roof and the façade of the spires and the interior like the wooden pipe organ or sculptures of the cathedral. The outdoor data acquisition was performed in GPS based flight mode in team operation. Each spire was recorded from bottom to top with overlapped images in a constant distance of approx. $12 \mathrm{~m}$ from all sides. The recording time for both spires was less than 2 hours. The data recording between both spires was the most critical part due to the short distance to the structure and the complex wind conditions (structure induced turbulences). The photos were used for aligning/generating rectified overview orthophoto mosaics of all side views for a first fast pre-check of the surface. Due to the high resolution of the several images the orthophotos allow a detailed check for even small damages on the surface. Fig. 3 top shows one sample image of these overview data recorded, left in original size and right the $100 \%$ resolution with detected damages on the slate roof. After analysing the overview orthophotos, critical parts with supposed or detected damages were investigated and recorded in detail with photos and videos in close proximity of only $3-5 \mathrm{~m}$ to the structure. Fig. 3 bottom shows thin cracks on the freestone masonry detected by detailed surface analysis. 

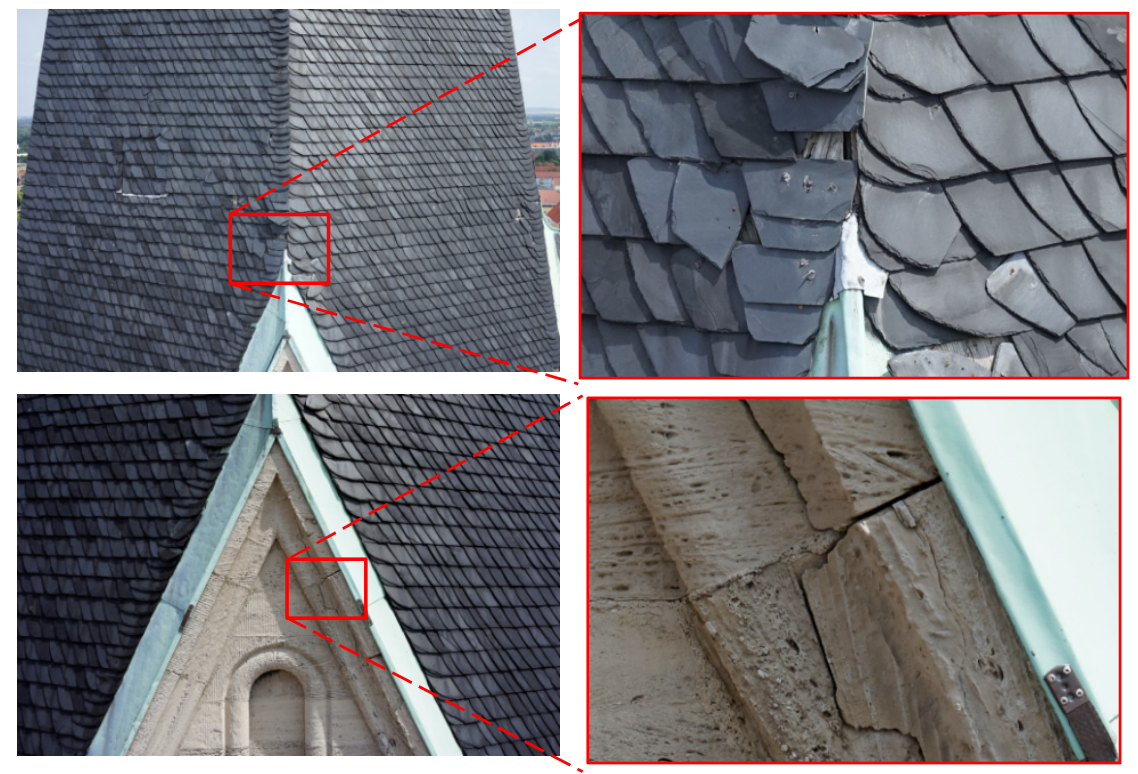

Figure 3: Roof and façade inspections results, left: original overview photo, right: detail of detected damages (100\% resolution), missing slate plates and cracked freestone bricks.

With the use of UAS parts like the top of the spire that are otherwise extremely difficult to access can be inspected very easily and in high quality such that also restorers and conservators get helpful information for their condition assessment and restauration planning. Fig. 4 shows one result of the top spire inspection.
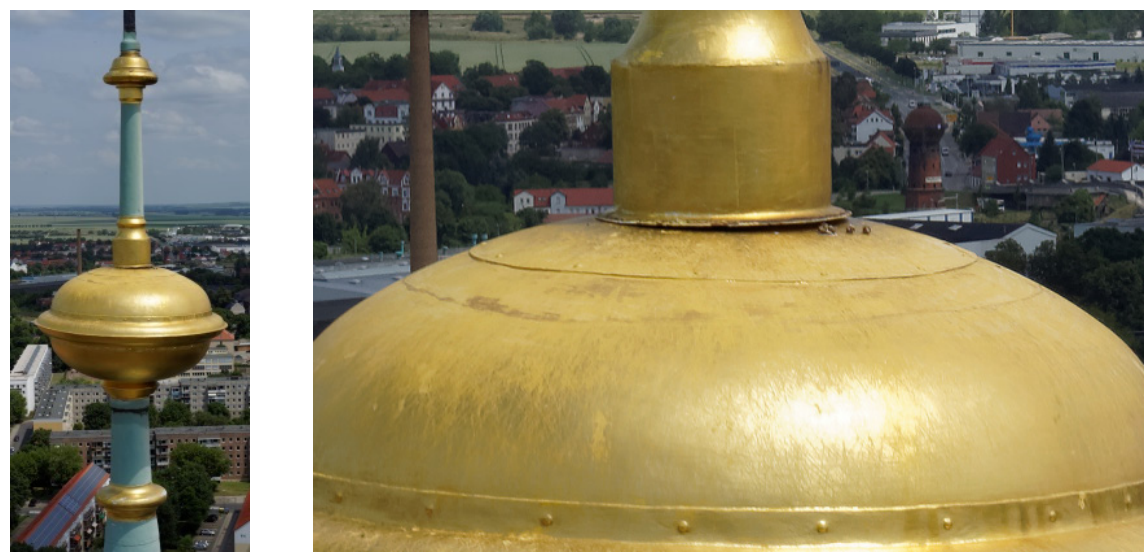

Figure 4: Spire inspection results: left sections of original photos, right: detail of detected damages (100\% resolution), loose connection (uplift between bowl and cross) and damaged gilded surface. 
The interior inspections were performed in altitude supported flight mode without any support of GPS. But the extreme flight stability of the systems allows also for flights in distances of less than $2 \mathrm{~m}$ to the structure. That helps to generate highly detailed images. By using interchangeable zoom lenses the parts of interest could recorded in higher degree of detail if it is required for the condition assessment. Additionally to the still image based inspections live-video inspection flights were performed outdoor and indoor with a fully stabilised camcorder mounted on the flight system. The live video was streamed on the ground control station and on a second screen such that all experts could inspect the structure together. By using the large optical zoom it was possible to detect tiniest damages and it was even possible to estimate the degree of pollution like dust or discolouration of restored surfaces.

\subsection{Aerial images based survey and damage detection}

The image quality shown in section 3.1 leads to the assumption that such images can be used for photogrammetry, survey and 3D reconstruction of structures to obtain information about geometry parameters or deformations of the structure. This application was tested on one of the most inclined historical towers of the world, the leaning tower of Bad Frankenhausen, shown in Fig. 5 (north view).

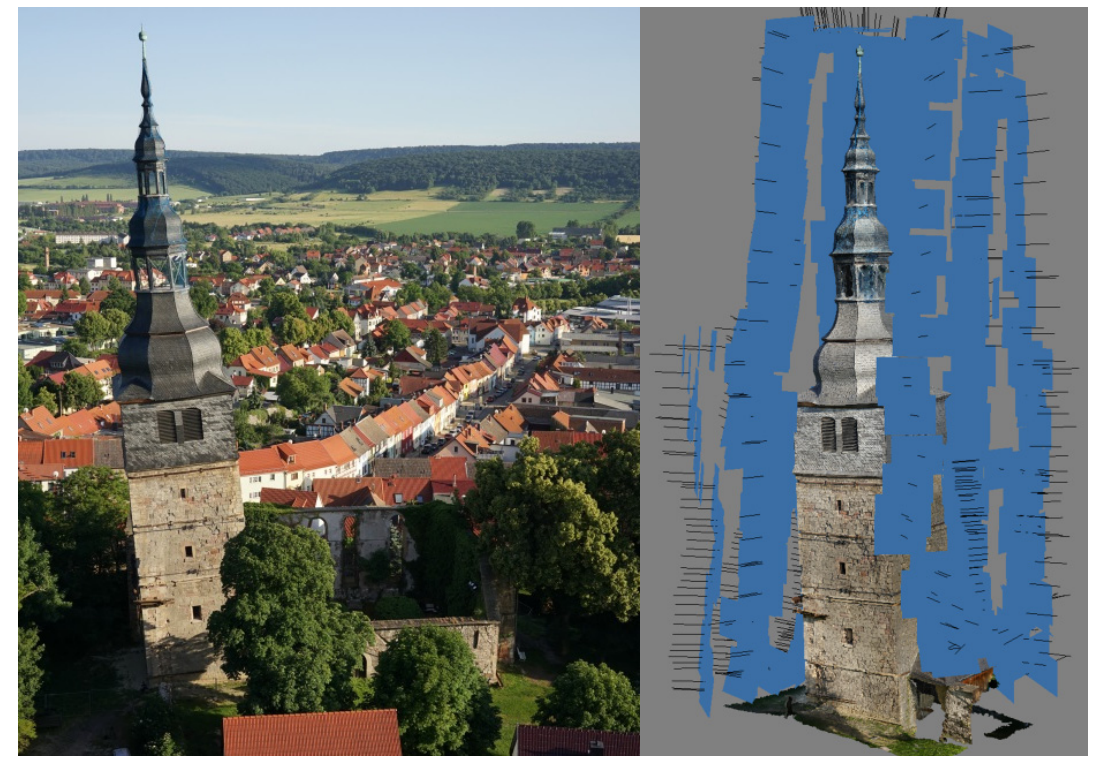

Figure 5: Left: leaning tower of Bad Frankenhausen (north view), right: reconstructed camera positions and flight path during data recording.

Due to the geological characteristics the inclination of this tower continuously increases very slowly. The movements of the tower cause deformations on the natural masonry additionally which can only be measured by recording and 
monitoring the tower surface in detail by using dense point clouds. Conventional methods like terrestrial laser scans are limited to record the complete structure, e.g. roofs or shaded areas. The use of UAS allow for a complete data capture all around the structure. The tower was recorded in approx. 90 minutes in eight vertical flight paths with overlapped images in vertical and horizontal direction. Additionally a circle flight with vertical camera tilt to the ground was performed to capture the invisible parts of the roof. The reconstructed flight paths with the estimated camera positions are shown in fig. 5 right. The 525 images of the tower were used for UAS-Photogrammetry [8] for a geo-referenced 3D reconstruction of the tower using modern computer vision methods like Structure-from-Motion (SfM). SfM methods are used to estimate the motion of a single camera and the 3D structure at the same time. This approach allows a highly flexible image acquisition process. In a first step suitable imagery of the overlapped images of the structure must be selected by hand for the reconstruction. Based on the Scale Invariant Feature Transform (SIFT) and the RANdom Sample Consenus (RANSAC) approach robust tie points between the images are automatically extracted, described and matched to detect corrospondence image pairs. The SfM algorithm is applied to recover the camera position and orientation parameters and the sparse 3D geometry. The Exif metadata were used to support the camarea calibration, e.g. approximation of the focal length. All estimated parameters including the non-linear distortions are refined using a Bundle Adjustment algorithm that globally minimizes the reprojection errors within the whole image scene. Finally, a dense stereo image matching algorithm is required to recover a continuous and detailed surface. At first, the optimized interior orientation parameters are used to remove radial lens distortion from the images. Typically, a fast multi-view stereo algorithm is applied to recover dense surface models. Starting with feature-based patches as support, a polygonal mesh is iteratively computed as dense and continuous representation of the scene. Fig. 6 shows the results of the dense 3D reconstruction in the steps of sparse and dense point cloud generation and the polygonal point cloud meshing for reconstruction of the tower surface.
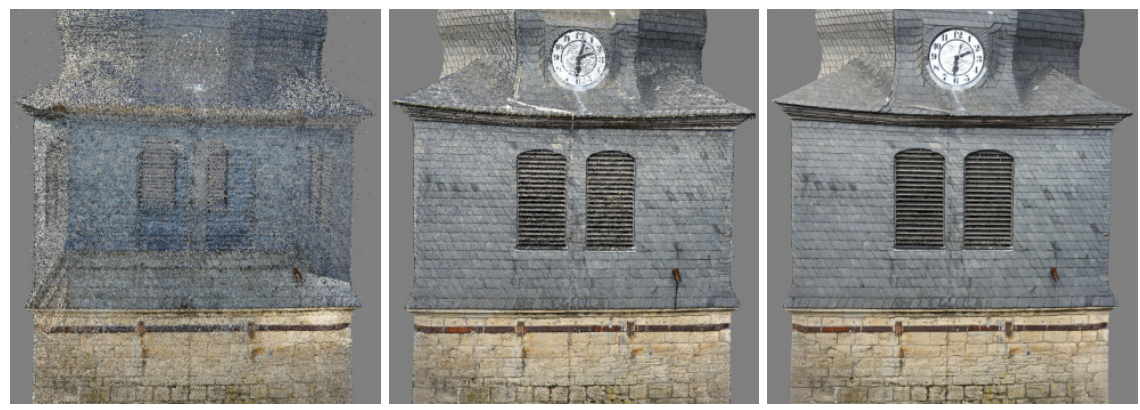

Figure 6: Detail of dense 3D reconstruction of the leaning tower using Structure-from-Motion (SfM), left: sparse point cloud (7 mio. points), centre: dense point cloud (300 mio. points), dense polygonal colour coded mesh of reconstructed surface (62 mio. faces). 
To extract referenced geometrical data from the 3D reconstruction of the tower 16 ground control points were placed well distributed over the structure and georeferenced by conventional tachometry. These ground control points could be identified and referenced in the high resolution images. The results of the 3D reconstruction are summarised in the following table 2 and fig. 7 show the geometrical data extraction for the global inclination and the deformations of a damaged masonry edge from the $3 \mathrm{D}$ reconstruction.

Table 2: Results of geo-referenced 3D reconstruction of the leaning tower of $\mathrm{Bad}$ Frankenhausen.

\begin{tabular}{|c|c|c|c|c|c|c|c|}
\hline \multicolumn{3}{|c|}{ Images } & $\begin{array}{c}\text { Ground control } \\
\text { points }\end{array}$ & \multicolumn{3}{c|}{ 3D reconstruction } \\
\hline No. & $\begin{array}{c}\text { Object } \\
\text { resolution }\end{array}$ & Error & No. & Error & $\begin{array}{c}\text { 3D- } \\
\text { resolution }\end{array}$ & $\begin{array}{c}\text { Dense point } \\
\text { cloud }\end{array}$ & Mesh \\
\hline 525 & $1.34 \mathrm{~mm}$ & $\varnothing 0.51$ pixel & 14 & $\varnothing 2.66 \mathrm{~mm}$ & $2.68 \mathrm{~mm}$ & 300 mio. & 62 mio. faces \\
\hline
\end{tabular}
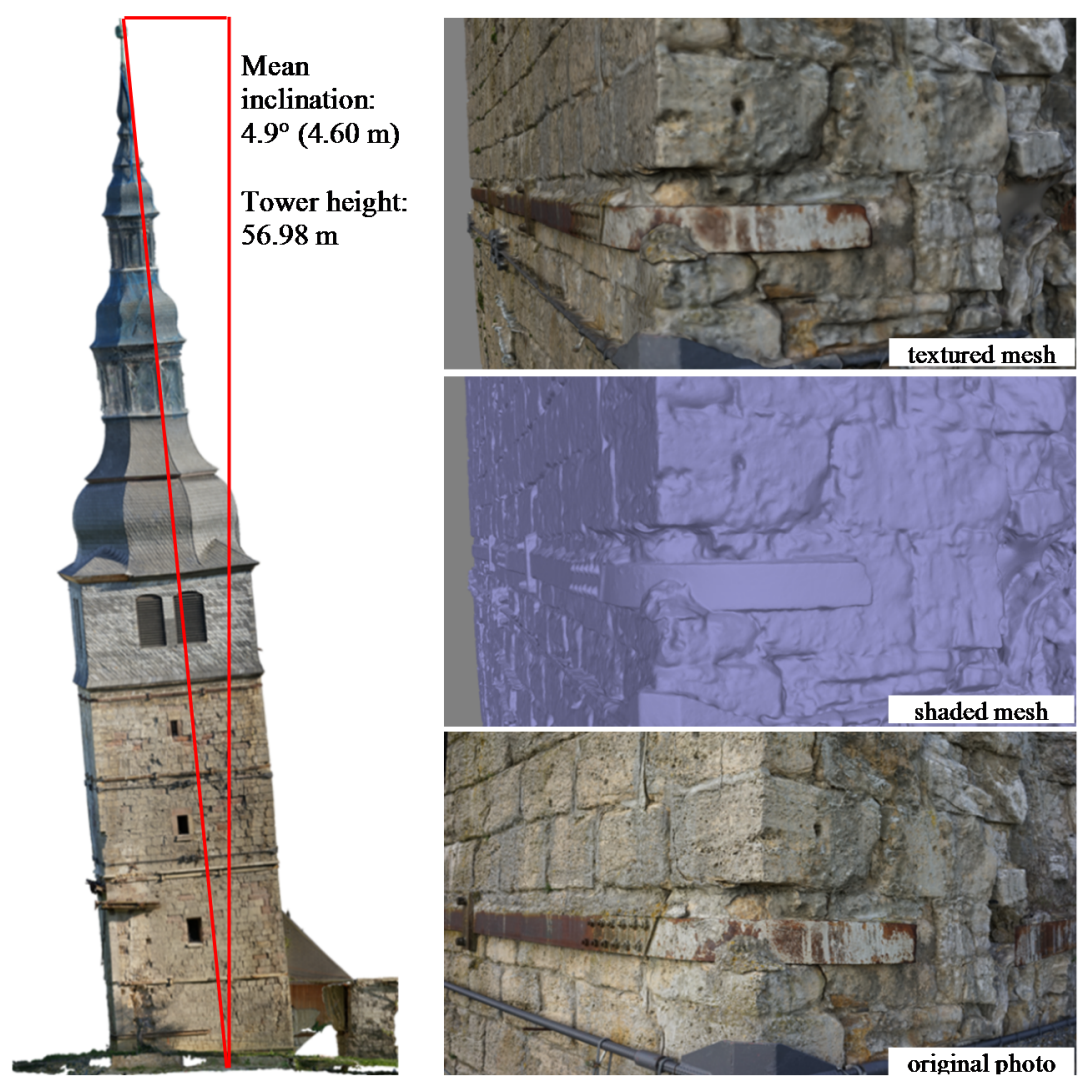

Figure 7: Left: orthophoto mosaic of the leaning tower (north view), right: detail of the dense $3 \mathrm{D}$ reconstruction of a damaged masonry edge. 
The generated geo-referenced dense point cloud can also be used for continuous motion and deformation monitoring of the tower. Using fixed ground control points next to the structure displacements and changes (deformations) on the structure can be detected and visualised by colour coded differential point clouds. The applications and test results of an automatic change detection monitoring workflow based on aerial images, geo-referenced 3D reconstruction and cloud comparison are shown and explained in detail in [5].

\subsection{Aerial image based high accurate 3D reconstruction of sensitive sculptures}

Sculptures or structural elements like the aesthetic gargoyles are very sensitive structural parts. The process of condition assessment is much more complex due to the special locations and the high sensitivity of those figures. The application of UAS based high detailed visual state determination and high accurate 3D reconstruction for maintenance or complex restorations was tested on the sculpture of St. Hieronymus in the Halberstadt Cathedral, shown in fig. 8. This sculpture hangs on an interior pier of the nave and was recorded in only one flight in 12 minutes by 180 images in a distance of less than $2 \mathrm{~m}$. The photos were taken in different camera angles for an entire capture of the sculpture, though the critical light conditions (darkness) lead to a challenging data acquisition procedure.
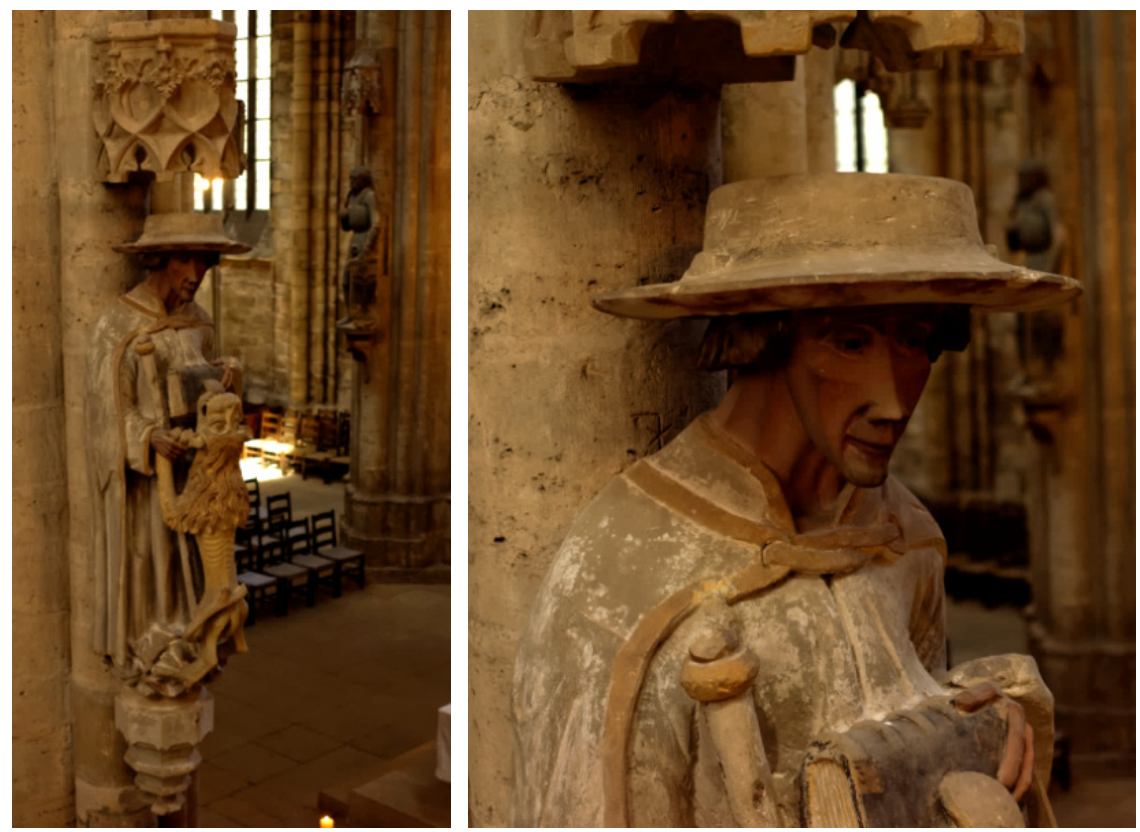

Figure 8: Image section of the original aerial photos of St. Hieronymus in Halberstadt Cathedral, Germany. 
The 180 images with an object resolution of approx. $0.28 \mathrm{~mm} /$ pixel were used for a true scale and coloured fidelity 3D reconstruction using SfM methods explained in section 3.2. Based on the high resolution and a post flight quality enhancement of the RAW-images an ultra-dense RGB point cloud with more than 43 million measurement points could be generated. Using the dense point cloud for polygonal meshing the fine surface of the sculpture could be modelled highly accurately with approx. 8.5 million faces. For visualisation the high quality images were used for a textured 3D model. Fig. 9 shows a section of the reconstructed 3D model, left with the meshed and textured surface and right with the polygonal meshed surface. The achieved accuracy allows for detailed visual and geometrical analysis of the entire structure. That also leads to the concept of 3D modelling of missing, broken or damaged parts of the sculpture which could be renewed/reconstructed partial or all complete by $3 \mathrm{D}$ prints in original size.

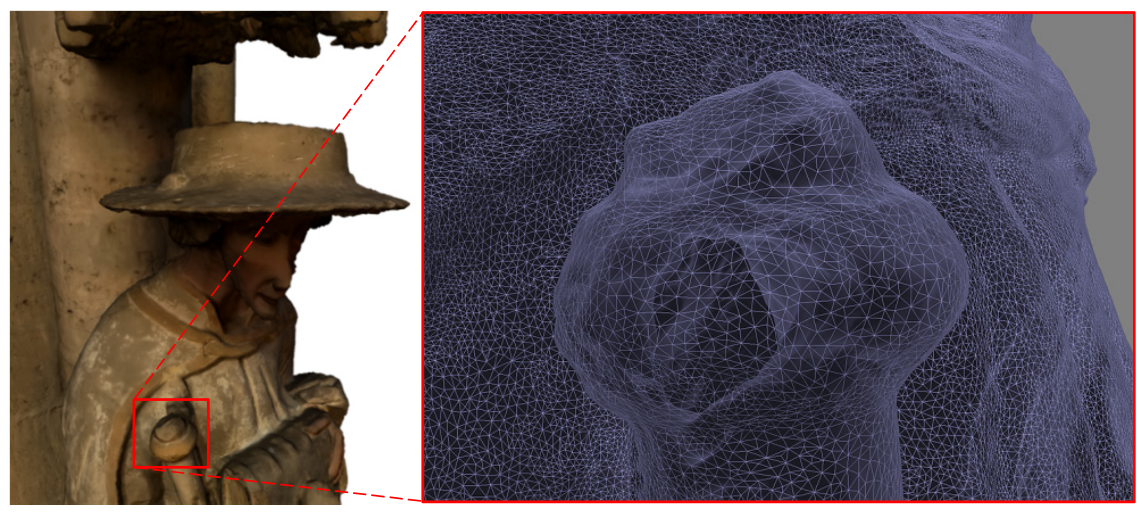

Figure 9: Left: colour fidelity dense 3D reconstruction of the sculpture (43 mio. points, 8.5 mio. faces), left: shaded polygonal meshed surface.

\section{Summary, conclusions and outlook}

This paper presented the capability and possible applications of professional highend remote controlled unmanned aerial systems in the field of preservation, inspection, survey and monitoring of heritage monuments based on high quality aerial images in combination with modern computer vision methods for structural analysis. The advanced flight systems provide a very fast, safe and potentially semi-autonomous data acquisition without using special access units like scaffoldings, elevating platforms or professional climbers. This avoids complex and protracted data acquisition processes that also often cause aesthetic disturbances. The high quality video and image data can be used for post flight analysis and detailed location-referenced documentation of the investigated structure.

This drastically increases the efficiency of inspection and monitoring of heritage monuments by saving time and money for data acquisition in comparison to conventional methods. 
The modern computer vision methods described allow the fully automatic generation of dense surface reconstructions from digital high quality imagery. The successful application tests on the leaning tower has shown that high quality aerial images together with georeferenced control points can be used for a photogrammetric analysis to perform 3D measurements for highly accurate survey. The tests on the sculpture have shown the potential of this UAS-based method for ultra-high accuracy and colour fidelity 3D reconstruction of high sensitive structures. It is easy to imagine that in the future such data could be used for the complete reconstruction of damaged sculptures or structural parts to replace them by $3 \mathrm{D}$ prints.

\section{Acknowledgements}

The authors gratefully acknowledge the technical support by Ascending Technologies and the support by Volker Lind (cathedral engineer of the Halberstadt Cathedral), Uwe Kalisch (Institut für Diagnostik und Konservierung an Denkmalen in Sachsen und Sachsen-Anhalt e.V.--IDK) and Ralph Lindemann (The Stiftung Dome und Schlösser in Sachsen-Anhalt).

\section{References}

[1] Hallermann, N. \& Morgenthal, G., The Application of Unmanned Aerial Vehicles for the Inspection of Structures, Proc. of PLSE 2012, Hong Kong, pp. 1085-1095, 2012.

[2] Hallermann, N. \& Morgenthal, G., Unmanned aerial vehicles (UAV) for the assessment of existing structures, Proc. of IABSE Symposium 2013, pp. 266267, Kolkata, 2013.

[3] Morgenthal G. \& Hallermann, N., Quality Assessment of Unmanned Aerial Vehicle (UAV) Based Visual Inspection of Structures. Advances in Structural Engineering, Volume 17 No. 3 2014, pp. 289-302, 2014.

[4] Hallermann N. \& Morgenthal G., Visual inspection strategies for large bridges using Unmanned Aerial Vehicles (UAV). Proc. of 7th IABMAS, International Conference on Bridge Maintenance, Safety and Management, pp. 661-667, Shanghai, 2014.

[5] Hallermann N., Morgenthal G. \& Rodehorst V., Vision-based deformation monitoring of large scale structures using Unmanned Aerial Systems. Proc. of 37th IABSE Symposium, Engineering for Progress, nature and People, pp. 2852-2859, Madrid, 2014.

[6] Ascending Technologies, www.asctec.de

[7] Fraunhofer IRB, https://www.irb.fraunhofer.de/bauforschung/baufolit/ projekt/Unbemannte-Flugger\% $\% 3 \% A 4$ te-zur-Zustandsermittlung-vonBauwerken/20138035900

[8] Eisenbeiß, H., UAV Photogrammetry. Dissertation, ETH Zurich, 2009. 